\title{
Effect on subsequent fixed-interval schedule performance of prior exposure to ratio and interval schedules of reinforcement
}

\author{
PHIL REED \\ Swansea University, Swansea, Wales \\ AND \\ Theresa A. Morgan \\ University of Iowa, Iowa City, Iowa
}

\begin{abstract}
In three experiments, we examined the effect on the patterns of responding noted on fixed interval (FI) schedules of prior exposure to a range of interval and ratio schedules. Rats leverpressed for food reinforcement on random ratio (RR), random interval (RI), or variable interval (VI) schedules prior to transfer to FI schedules. In Experiment 1, prior exposure to an RR schedule retarded the development of typical FI patterns of responding. Exposure to a yoked RI schedule produced even greater retardation of typical FI performance. This effect was replicated in Experiment 2, using a within-subjects design. Rats responded on a multiple RR-RI schedule prior to a multiple FI-FI schedule. Typical FI performance emerged more slowly in the component previously associated with the RI than with that associated with the RR. In Experiment 3, exposure to an RR schedule retarded the development of FI performance to a greater extent than did exposure to a VR schedule. The latter schedule was programmed to allow the possibility that inhibitory control would develop after reinforcement. These results confirm that ratio schedules independently result in the disruption of FI responding. This effect was not long lasting and cannot be used plausibly to explain species differences in responding to FI schedules. However, it does suggest that temporal control - as manifested by the transfer of inhibitory control from one schedule to another - could facilitate movement between interval schedules.
\end{abstract}

Although focus on the impact of currently operative contingencies on behavior is common, many investigators have also shown the importance of the effects of historical training on current behavior. Behavioral history effects are said to occur when previously learned response topographies - which are no longer reinforced-nonetheless continue to exert control over current behavior (Tatham \& Wanchisen, 1998). Two commonly referenced examples of history effects include the "resurgence" of previously reinforced but currently extinguished responses (see, e.g., Mechner, Hyten, Field, \& Madden, 1997; Reed \& Morgan, 2006, 2007) and the "reinstatement" of previously reinforced but currently extinguished responding due to response-independent reinforcement (Doughty, Reed, \& Lattal, 2004). In both examples, a previously reinforced response - a simple response, like a leverpress (Doughty et al., 2004); a more complex response, like a sequence of responding (Mechner \& Jones, 2003); or even a pattern of responding generated by a schedule of reinforcement (Reed \& Morgan, 2006) - persists in the current performance of an organism, despite its not being currently programmed for reinforcement. In the latter example, rats with scheduled-interval and ratio-historical training showed dif- ferential rates of response in extinction, despite intervening interval training that produced equitable response rates.

One procedure that has been employed a number of times to investigate schedule history effects examines the effect of prior conditioning history with variable ratio (VR) schedules on subsequent fixed interval (FI) performance. Weiner $(1964,1969)$ noted an enduring effect of training with VR schedules on human FI performance, as compared with that performance in the absence of such training. This effect was also reported in rats by Wanchisen, Tatham, and Mooney (1989). In the latter study, rats responded for 30 sessions on a VR-20 schedule prior to responding on 40 sessions on an FI 30 -sec schedule. In comparison with a group that responded only on the FI schedule, rats in the first condition showed very little evidence of either response scalloping (see Ferster \& Skinner, 1957) or break-and-run responding (Dews, 1978) on the FI schedule. Instead, the rats tended to emit either high or low rates of responding on the FI schedule.

There are two issues connected to this specific behavioral history literature that require further investigation prior to the development of theory. First, although experimenters investigating the effects of transfer from VR to FI

P. Reed, p.reed@swansea.ac.uk 
schedules were able to control for the rate of reinforcement in movement from one schedule to another, the effects of prior rate of reinforcement between groups (e.g., the history VR group and the control group) were not equated. There is considerable debate in the resurgence literature concerning the relative importance of the rate of response or reinforcement in determining such behavioral history effects (see Doughty et al., 2004; Reed \& Morgan, 2007). In establishing the parameters necessary for such history effects, this would appear to be an important variable to study.

Second, there are two sets of nonoverlapping studies in the literature. Although both focus on the effects of prior exposure to various schedules on the development of FI responding, the previously mentioned studies used ratio-based schedules that were response dependent (e.g., Baron \& Leibenweber, 1995; Wanchisen et al., 1989), whereas earlier studies focused on the impact of timebased schedules that were response independent (e.g., Trapold, Carlson, \& Myers, 1965). Trapold et al. showed that, in comparison with variable time (VT) exposure, previous exposure to fixed time (FT) schedules facilitated subsequent transfer to FI schedules. In this study, groups of rats given FT pretraining more easily displayed classic scalloping response patterns on an FI 120-sec schedule than did VT pretrained groups or groups with no pretraining. One suggested hypothesis for the differential response patterns resulting from such transfer focuses on the issue of temporal control. That is, FT and FI schedules share elements of temporal control over scheduled reinforcement. In relation to the above schedule history effects (see, e.g., Wanchisen et al., 1989), it could be argued that movement from ratio to interval schedules would produce no such facilitation. In fact, it may lead to retardation of transfer, but this point was not discussed by Trapold et al.

An obvious difference between these earlier studies (e.g., Trapold et al., 1965) and the later VR to FI reports (e.g., Wanchisen et al., 1989) is that the former employed response-independent reinforcement schedules, whereas the latter employed response-dependent reinforcement schedules. Of course, such schedules would differ on more than their temporal characteristics (and, to some extent, this issue overlaps with the issues of the importance of response rate and pattern in the history building schedule discussed previously). Thus, the explanation of temporal control as the sole avenue for easy transfer between schedules is not necessitated by data presented by Trapold et al. Both FT and FI schedules are characterized by a long postreinforcement pause (although there are clearly many other differences between the performance engendered by the two schedules). As such, transfer between schedules with this pattern of behavior may appear to occur more quickly because of similar response patterns being involved, rather than because of learned temporal control (see Keenan \& Leslie, 1986). Lopez and Menez (2005) compared response patterns on subsequent FI schedules for rats given continuous reinforcement (CRF), FT, and random interval (RI) pretraining. The results of this study suggested that facilitated transfer to an FI pattern occurs due to both temporal allocation of responses within the interval and simple response output. As such, it remains unclear whether the transfer of learning is an issue of temporal control or one of simple similarity in the patterns of behavior produced by the schedules (both history and transfer).

Thus, the present study sought to address these two areas of ambiguity in the behavioral history literature relating to the effects of prior histories prior to transfer to FI schedules. First, we aimed to explore the possible impact of differential reinforcement rates across the history building schedules. Second, we aimed to partially reconcile this literature by investigating the effect of prior exposure to either time-based or response-based response-dependent schedules on FI performance. The resolution of some of these issues should provide a firmer foundation for the development of subsequent theorizing in this area.

\section{EXPERIMENT 1}

The first experiment examined the impact of previous training with either random ratio (RR) or yoked RI schedules on subjects' subsequent FI performance, as compared with that of subjects who were not exposed to these schedules previously. The RI schedule would have a lower response rate than the RR schedule; however, it would have the same rate and pattern of reinforcement as would the RR schedule, because of a yoking procedure. In this way, we hoped to explore whether the rate of responding or the rate of reinforcement determined the strength of the history effect. Moreover, these data might allow a test of the temporal-based hypothesis. To the extent that subjects learn that a contingency is time based (RI), they may transfer more readily to another time-based schedule (FI) than they would when the history building contingency is response based (VR). Although there is clearly a difference between RI and FI schedules in that the temporal contingency of one is fixed and that of the other is random, there is evidence of learning about the temporal nature of the RI schedule (Roberts, Tarpy, \& Lea, 1984), which should facilitate transfer to an FI schedule-certainly better than to a VR schedule.

\section{Method}

Subjects. Twenty-four experimentally naive male Lister hooded rats served in the present experiment. The subjects were 3-4 months old at the start of training, had a free-feeding body-weight range of $350-445 \mathrm{~g}$, and were maintained at $85 \%$ of this weight throughout the experiment. The animals were housed in groups of 4 , with water constantly available in the home cage.

Apparatus. Four identical operant conditioning chambers (Campden Instruments Ltd.) were used. Each chamber measured $23.5 \times$ $23.5 \times 20.5 \mathrm{~cm}$ and was housed in a light- and sound-attenuating case, ventilated by a fan that provided background masking noise (65 dB[A]). Each chamber had two levers, the left of which was permanently inserted into the chamber. Reinforcement consisted of 45-mg standard Noyes food pellets, which were delivered to a centrally located, recessed food tray that was covered by a clear Perspex hinged flap. The chamber was not illuminated during the course of the experiment

Procedure. The subjects were magazine trained in two 20 -min sessions of a random time (RT) schedule. They then received leverpress training using two 20-min sessions of a CRF schedule. Subjects in Group RR received training according to a schedule that had a constant probability of reinforcement for every response emitted (e.g., on an RR-5 schedule, the probability of reinforcement for 
every response was .20 ). The mean value for the RR schedule was increased as follows: Session 1, RR-5; Session 2, RR-10; Sessions 3-4, RR-15; Sessions 5-6, RR-20; Sessions 7-8, RR-25; Sessions 9-15, RR-30; Sessions 16-20, RR-45; and Sessions 16-30, RR-60. Subjects in Group RI were yoked to the subjects in Group RR on a one-to-one basis. The same sets of two animals (one in Group RR and one in Group RI) remained paired throughout the study. The value of the interval criterion was determined on a reinforcer-toreinforcer basis by the time taken to earn a food pellet by the master rat in Group RR. Sessions lasted for $60 \mathrm{~min}$ each. The remaining group of animals (Group None) received magazine and leverpress training only.

During the second phase of training, all subjects were placed on an FI 60-sec schedule of reinforcement for 20 sessions, each lasting $60 \mathrm{~min}$.

\section{Results and Discussion}

Following the first phase of training with the RR and RI schedules, the mean total number of responses emitted during the final session for Group RR was 2,477 (82.5 responses/min), and this score was 1,138 (37.9 responses/ $\mathrm{min}$ ) for Group RI. This difference in the level of responding reflects the typical response-rate difference seen during the course of training and that which occurs when such yoking procedures are adopted (Ferster \& Skinner, 1957).

The top panel of Figure 1 shows the mean responses emitted for each group during each successive 6-sec interval of the FI schedule, over the four 5-session blocks of training. These data were analyzed by a three-factor mixed-model ANOVA, with schedule (RR vs. RI vs. none) as a between-subjects factor and session and interval as within-subjects factors. A rejection criterion of $p<.05$ was adopted for this and all subsequent analyses. The data and statistical analysis show that there was a difference in the rate of response between the groups, with Group RR responding faster than did Group RI, which, in turn, responded faster than did Group None [main effect of schedule, $F(2,19)=42.63]$. Responding declined over
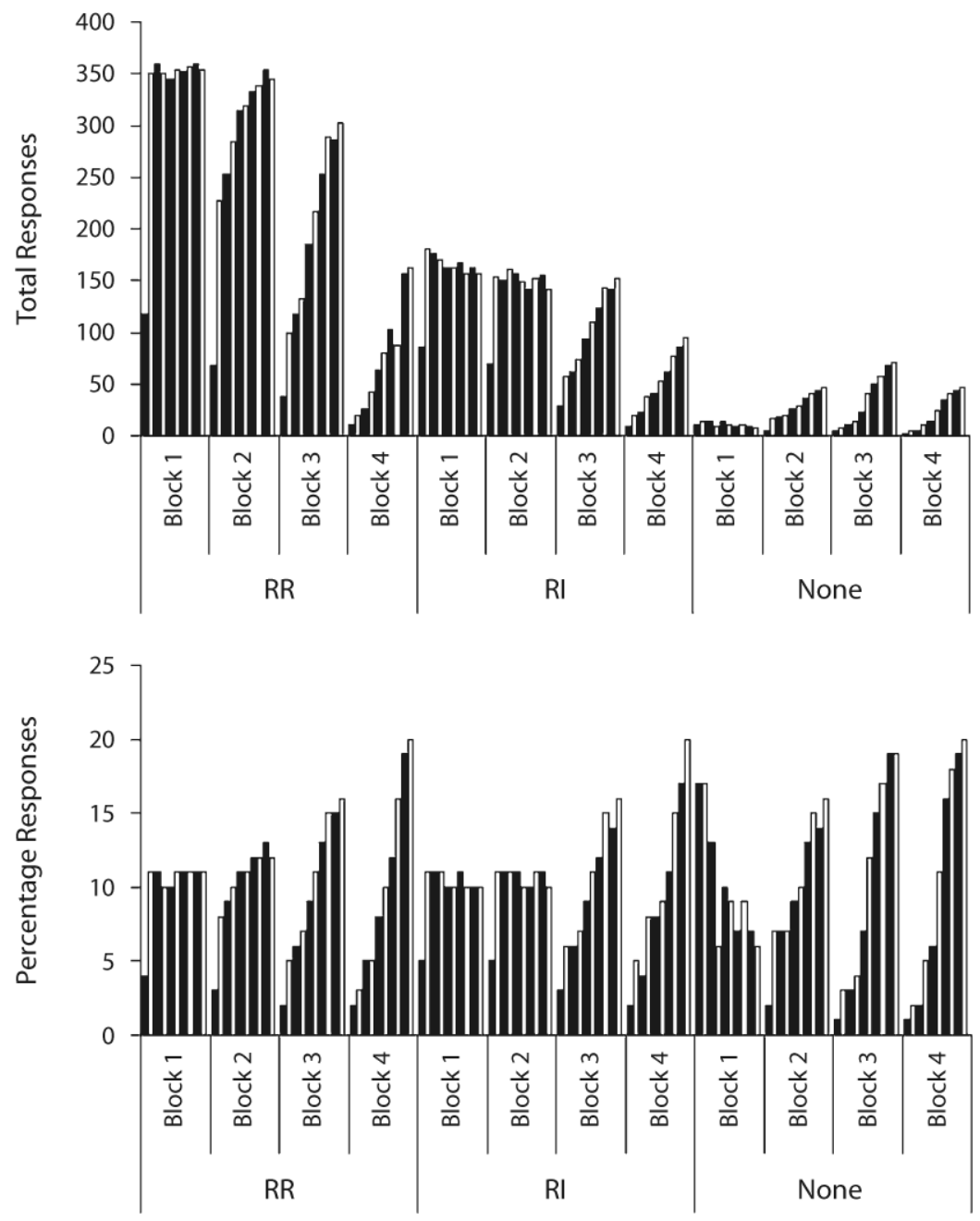

Figure 1. Results from Experiment 1. Top panel: Mean number of responses emitted in each 6-sec interval during exposure to the FI 60-sec schedule over the four 5-session blocks of training. Bottom panel: Mean percentage of total responses emitted during each successive 6-sec interval of the FI 60-sec schedule. RR, prior training on a random ratio schedule; RI, prior training on a random interval schedule; None, no prior training. 
the course of training [main effect of session, $F(3,57)=$ 17.69], and responding altered across the successive intervals of the FI schedule [main effect of interval, $F(9,171)=$ 50.99]. Inspection of these data also shows a variety of interactions between the groups across the intervals, which changed over the course of training [three-way interaction, $F(54,513)=3.14]$.

The three-way interaction was analyzed by a series of two-factor ANOVAs (group vs. interval) conducted on each 5 -session block, as recommended by Howell (1997). Inspection and analyses of the data for Sessions 1-5 show that there was a difference between the responding due to pretraining $[F(2,19)=46.55]$, that there were differences across the intervals $[F(9,171)=69.13]$, and that the pattern across the intervals differed between the groups $[F(18,171)=27.90]$. For Group RR, there was a low rate immediately after reinforcement, followed by a fairly constant high rate across the interval [linear trend, $F(1,6)=$ 31.36; quadratic trend, $F(1,6)=43.28]$. For Group RI, there was a slightly smaller decline in responding following reinforcement, followed by an increase, then by a gradual decrease in responding across the rest of the FI interval [linear trend, $F(1,6)=11.73$; quadratic trend, $F(1,6)=81.55]$. There was no clear pattern of responding in the FI condition (both linear and quadratic $p \mathrm{~s}>.30$ ). These data are consistent with the patterns of responding to be expected on the basis of previous training. Responding on an RR schedule across a trial would be reflected in a slight pause - proportional to the size of the RRfollowed by constant high rates (Dembo, Becerra de Penfold, Ruiz, \& Casalta, 1985). On an RI schedule, the pause would be slightly shorter than that on an RR schedule, with a slightly negative trend across the rest of the interval (see Toal \& Leslie, 1984). For Group FI, no pattern had emerged, due to insufficient exposure to the schedules.

During the second 5 -session block of training on the FI schedule, there remained a difference between the responding due to pretraining $[F(2,19)=24.67]$, there were differences in responding across the intervals $[F(9,171)=$ 36.69], and the pattern of responding across the intervals differed between the groups $[F(18,171)=13.25]$. For Group RR, there was a rate change across the intervals similar to that in the first 5-session block of training, but there was also a clearer positive increase in rate across the session [linear trend, $F(1,6)=39.02$; quadratic trend, $F(1,6)=13.08]$. For the RI group, there was a slightly smaller decline in responding following reinforcement, followed by a constant rate across the schedule [linear trend, $F(1,6)=10.07$; quadratic trend, $F(1,6)=9.77]$. For the FI schedule, a constantly increasing rate was observed across the intervals [linear trend, $F(1,7)=14.04$; quadratic trend, $F<1$ ].

During the third block of training on the FI schedule, there remained a difference between the responding due to pretraining $[F(2,19)=9.98]$, there were differences in responding across the intervals $[F(9,171)=37.96]$, and the pattern of responding across the intervals was different between the groups $[F(18,171)=5.25]$. For both Groups RR and RI, there was a clear positive increase in rate across the session [both linear trends were statistically significant, smallest $F(1,6)=16.37$; neither quadratic trend was statistically significant, both $p \mathrm{~s}>.10]$. For the FI group, there was a developing scalloped pattern, with responding being more pronounced in the latter portion of the interval [linear trend, $F(1,7)=27.21$; quadratic trend, $F(1,7)=9.34]$.

During the final 5 -session block of training, there was little effect of pretraining $(p>.10)$ and little differential responding between the groups across the intervals $(p>$ $.05)$. All groups developed a similar pattern of scalloped responding $[F(9,171)=14.30$; linear trend, $F(1,19)=$ 19.53; quadratic trend, $F(1,19)=8.27]$.

An inspection of the percentage of responses emitted in each successive bin (bottom panel of Figure 1) allows the effect of pretraining to be seen in the absence of differences in overall response rate. These data show the slightly retarded development of a scallop pattern in Group RI as compared with that in Group RR. An analysis of the percentage of responses emitted during the first 6-sec interval-where this effect is most apparent-shows significant differences between the groups on each of the first three 5 -session blocks [smallest $F(2,19)=3.95$ ]. Tukey's honestly significant difference (HSD) tests conducted on these three blocks showed that for the first 5 -session block of training, Group None differed from each of the other two groups. For the second and third 5-session blocks, Group RI differed from Group None, but Group RR did not differ from Group None.

In sum, these data show that there was an effect of ratio pretraining on the rates of responding emitted during exposure to the FI schedule. Higher rates were maintained in pretrained subjects than in the non-pretrained subjects. However, this effect of previous ratio schedule training was relatively short-lived and had disappeared by the fourth 5-session block of training. The present data also replicated the effect of ratio pretraining on the subsequent FI pattern of responding. The subjects took longer to display the FI scalloped pattern after RR pretraining than after no pretraining. Thus, the results of Wanchisen et al. (1989) are partially replicated (although the effect was not long lasting).

A novel finding that emerged from the present experiment was that prior exposure to an interval schedule similarly seemed to retard the development of FI-like performance. In fact, if anything, this effect was stronger than that generated by previous exposure to a ratio schedule. This effect would not be predicted by the temporal hypothesis offered by Trapold et al. (1965). This view would predict faster transfer to typical FI patterns on the basis of learning that reinforcement was based on some temporal rule. Although certainly not as clear as that on an FT schedule, there should be more learning of temporal dependencies on an RI than on an RR schedule (see Roberts et al., 1984). Thus, a strong version of Trapold et al.'s temporal-based hypothesis would predict faster transfer from the former than from the latter pretraining schedule. However, response patterns on the FI schedule in the group that had been previously exposed to the RI schedule took longer to approximate those seen in the group with no training than did those noted in the group that had been 
pretrained with the RR schedule. In particular, it was seen that the subjects who were pretrained on the RI schedule did not pause for as long as those in the other two groups following reinforcement.

\section{EXPERIMENT 2}

Prior to further discussion of these results, and given the novel finding that emerged with respect to the effect of previous training with an RI schedule on FI performance, it was thought prudent to replicate the effects found in Experiment 1. This was believed important due to a potential effect of between-subjects yoking on performance. Church (1964) pointed out that yoking between subjects engenders the possibility of response-rate differences being introduced because of individual differences in sensitivity to reinforcement. To overcome this problem, a direct comparison was made between subjects' performance on FI components of a multiple FI-FI schedule when they had been pretrained on a multiple RR-RI schedule. The discriminative stimuli associated with each of the FI components were not changed from pretraining, as was the case in the study by Freeman and Lattal (1992).

Should the results of Experiment 1 be replicated, then the pattern of responding on the FI component previously associated with the RI schedule should take longer to show scalloped responding (and a shorter pause from responding) than would that associated with the RR schedule.

\section{Method}

Subjects and Apparatus. Eight experimentally naive male Lister hooded rats participated in Experiment 2. All of the rats were from 4 to 5 months old at the start of training, had a free-feeding bodyweight range of $360-420 \mathrm{~g}$, and were maintained as was described in Experiment 1. The apparatus was the same as that described in Experiment 1, with the exception that both levers were permanently inserted in the conditioning chamber.

Procedure. All rats received magazine training in two 20-min RT 60 -sec sessions. Following this, the rats were taught to leverpress in two 20-min CRF sessions. During the first session, the left lever was inserted into the chamber, and during the second session, the right lever was inserted into the chamber. The rats were then moved to a multiple CRF-CRF schedule for two 30-min sessions. During these sessions, the light above one lever would be illuminated for $5 \mathrm{~min}$. During this time, responses to that lever, but not to the other lever, would be reinforced. There would then be an intercomponent interval of $30 \mathrm{sec}$, during which all lights were extinguished. Following this period, the light above the other lever would be illuminated for $5 \mathrm{~min}$, and the reinforcement would be presented contingent upon pressing the lever, but not for pressing the other lever. This was repeated 12 times during the sessions (i.e., a total of six exposures to each lever occurred).

During the next sessions, the rats were all exposed to a multiple RR-RI schedule (half with the RR schedule on the right lever and half with the RR schedule on the left lever). The components of the multiple schedule were presented in strict alternation, with the mean interreinforcement interval on the RR schedule becoming the mean interval criterion for the RI schedule in the subsequent component. The RR criterion value was increased during this period as follows: Sessions 3-4, RR-5; Sessions 5-6, RR-10; Sessions 7-8, RR-15; Sessions 9-10, RR-20; Sessions 11-12, RR-25; Sessions 13-20, RR-30; Sessions 21-30, RR-40; Sessions 31-40, RR-50; and Sessions 41-60, RR-60. As noted above, each session com- prised six 5-min exposures to the RR and six 5-min exposures to the RI components.

Following exposure to this pretraining, the rats were all transferred to a multiple FI 60 -sec FI 60 -sec schedule. This training continued for 20 sessions. During each session, there were twelve 5-min components, six to each lever as described above. The stimuli associated with each component were unchanged from the history training.

\section{Results and Discussion}

Following the first phase of training with the RR and RI schedules, the mean total number of responses emitted during the final session for the RR component was 2,856 (86.2 responses/min), and this score was 1,045 (34.8 responses/min) for the RI component.

The top panel of Figure 2 shows the mean responses emitted for each component during each successive 6-sec interval of the FI schedule over the four 5-session blocks of training. These data are replotted as percentages of the total responses emitted in each 6-sec interval for the two components in the right panel of Figure 2. The response data (top panel) were analyzed by a three-factor, repeated measures ANOVA, with schedule (RR vs. RI), 5-session block, and interval as within-subjects factors. The data and statistical analysis show that there was a difference in the rate of response between components; the component previously associated with the RR schedule produced faster responding than did that previously associated with the RI schedule [main effect of schedule, $F(1,6)=25.29$ ]. Responding declined over the course of training [main effect of block, $F(3,18)=14.51]$, and responding altered across the intervals [main effect of interval, $F(9,54)=$ 22.14]. Inspection of these data also shows a variety of interactions between the components across the intervals, which changed over the course of training [three-way interaction, $F(27,162)=2.19]$.

The three-way interaction was analyzed by a series of two-factor ANOVAs (component $\times$ interval) that were conducted on each 5-session block. Inspection and analyses of the data for Sessions 1-5 show that there was a difference between the responding due to pretraining $[F(1,6)=30.46]$, that there were differences across the intervals $[F(9,54)=12.98]$, and that the pattern across the intervals differed between the components $[F(9,54)=$ 16.09]. For the RR component, there was a low rate immediately after reinforcement, followed by a fairly constant high rate across the interval [linear trend, $F(1,6)=19.27$; quadratic trend, $F(1,6)=40.73]$. For the RI component, there was a slightly smaller decline in responding following reinforcement, followed by an increase, then by a gradual decrease in responding across the rest of the FI interval [linear trend, $p>.05$; quadratic trend, $F(1,6)=$ 5.73]. These data replicate those found in Experiment 1 and are consistent with the patterns of responding to be expected on the basis of previous training.

During the second 5-session block of training on the FI schedule, there remained a difference between the responding due to pretraining $[F(1,6)=31.76]$, there were differences in responding across the intervals $[F(9,54)=$ 9.22], and the pattern of responding across the intervals 
differed between the components $[F(9,54)=7.89]$. For the RR component, there was a rate change across the intervals similar to that in the first block of training, but there was also a clearer positive increase in rate across the session [linear trend, $F(1,6)=71.03$; quadratic trend, $F(1,6)=23.64]$. For the RI component, there was a slightly smaller decline in responding following reinforcement, followed by a declining rate across the schedule [linear trend, $F<1$; quadratic trend, $F(1,6)=6.50$ ].

During the third 5-session block of training on the FI schedule, there remained a difference between the responding due to pretraining $[F(1,6)=6.40]$; there were differences in responding across the intervals $[F(9,54)=15.49]$, and the pattern of responding across the intervals was different between the components $[F(9,54)=3.88]$. For both the $\mathrm{RR}$ and RI components, there was a clear positive increase in rate across the session [both linear trends were statistically significant, smallest $F(1,6)=13.47$; neither quadratic trend was statistically significant, both $p$ s $>.30]$.
During the final 5 -session block of training, there still remained an effect of pretraining $[F(1,6)=9.14]$; there was a difference across intervals $[F(9,54)=14.78]$ and a difference in the pattern of responding across the trials, with a more scalloped pattern in the RR than in the RI pretrained component $[F(9,54)=4.16]$. For the RR component, the linear trend was significant $[F(1,6)=12.18]$, as was the quadratic trend $[F(1,6)=12.78]$. However, only the linear trend was significant in the RI component $[F(1,6)=24.38]$.

The key aspects of the results reported in Experiment 1 were replicated in Experiment 2, but we used a withinsubjects design instead of a between-subjects design. The key results are that pretraining does make a difference to the overall rate of responding when subjects are exposed subsequently to an FI schedule. This effect dissipates as exposure to the FI schedule continues, which is not surprising; however, it does support the data reported by Freeman and Lattal (1992) and by Wanchisen et al. (1989).
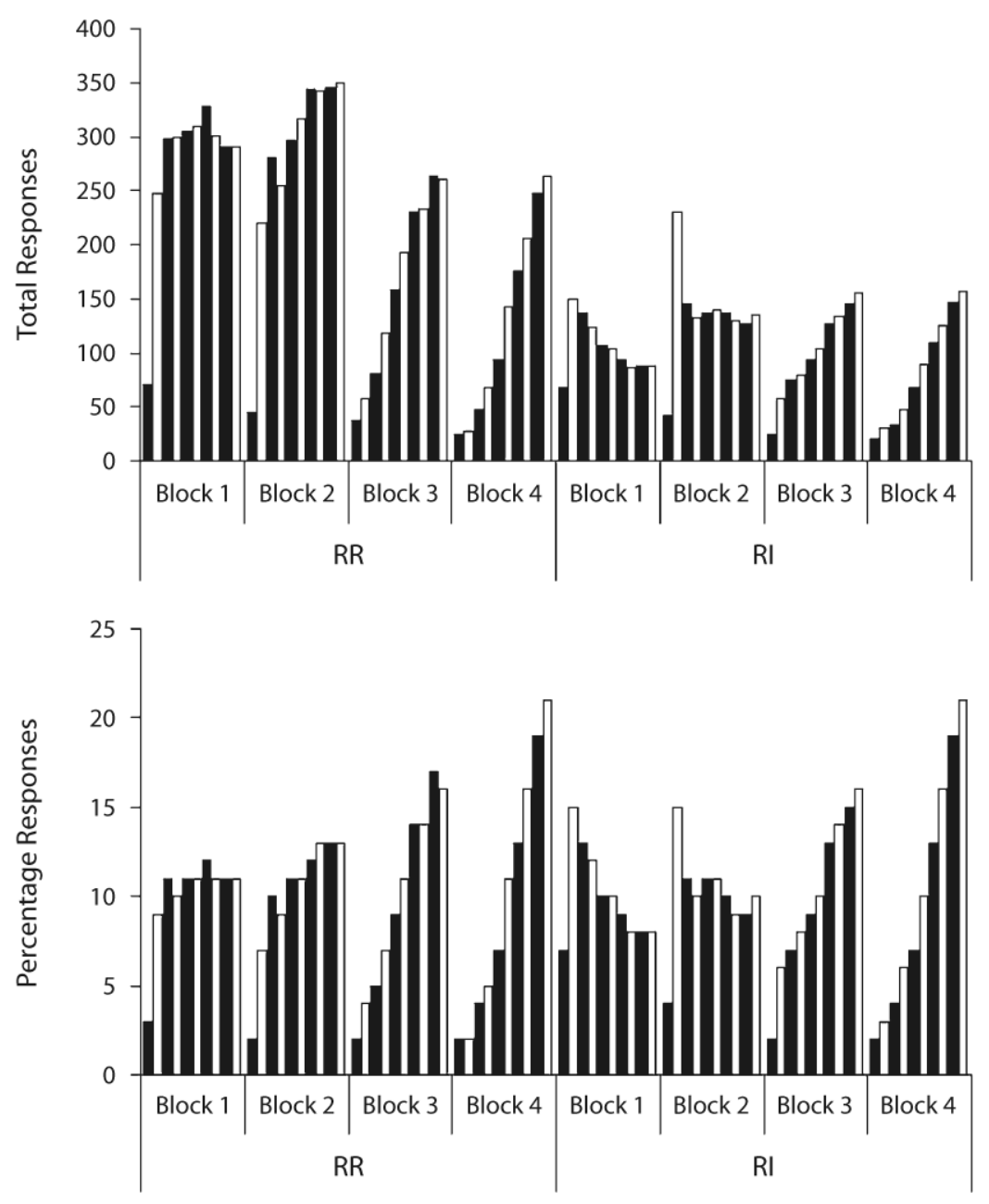

Figure 2. Results from Experiment 2. Top panel: Mean number of responses emitted in each 6-sec interval during exposure to the multiple FI 60-sec FI 60-sec schedule over the four 5-session blocks of training. Bottom panel: Mean percentage of total responses emitted during each successive 6-sec interval of the multiple FI 60-sec FI 60-sec schedule. RR, prior training on a random ratio schedule; RI, prior training on a random interval schedule. 
More importantly for the present purposes, the present data support the finding that prior exposure to a RR schedule does not hinder the development of responding typical of an FI schedule as much as does prior exposure to an RI schedule. The main effect of exposure to an RI schedule appears to be one of preventing the development of long pauses from responding immediately after the delivery of reinforcement while responding on the FI schedule. This can be seen most clearly in the bottom panel of Figure 2. These data show a greater percentage of responses emitted immediately after reinforcement in the RI as compared with the RR component - an effect that was statistically significant for the first and second 5-session blocks of training [smallest $t(6)=3.23$ ]

A possible factor responsible for the generation of the difference between the RR and RI schedules is their differing rates of response prior to the transition to the FI schedule. Another possible factor is the degree to which the schedules might allow the delivery of reinforcement to generate inhibitory control over responding. It is well established that rates of response immediately following reinforcement on, for example, an RR schedule are slightly lower than those following reinforcement on RI schedules (Toal \& Leslie, 1984; Zeiler, 1977). It is possible that this result is due to the fact that the first response following reinforcement may well be reinforced quite often on an RI schedule, but that this is less likely on an RR schedule. If this were the case, then the delivery of reinforcement would come to act as an inhibitory cue (an S- $\Delta$ ) for responding on the RR schedule, but not on the RI schedule. Such inhibitory properties may facilitate transfer to, for example, an FI schedule, which also produces pronounced pauses following reinforcement because of inhibitory properties of the reinforcement (Dews, 1962).

\section{EXPERIMENT 3}

The preceding two experiments showed a difference in the development of FI-typical responding, depending on whether pretraining was given on an RI or an RR schedule. This difference could not be due to the rate at which reinforcement was delivered on the two schedules but may have been due to differences in the rate of response or the potential for differences in inhibitory control to develop by the reinforcer. One possible method of unpicking the latter two possibilities is to examine the difference between prior exposure to both an RR schedule and a VR schedule. These schedules can be programmed to generate the same rates of response (having the same mean values), but the VR schedule - as opposed to the RR schedule with a constant probability of reinforcement for each response - can be programmed never to reinforce responding for the response immediately following reinforcement. This should generate inhibitory control on the VR schedule (see Toal \& Leslie, 1984, for a similar argument concerning VI and $\mathrm{RI}$ schedules). If the VR schedule generates slightly more inhibitory control than would the RR schedule, then it should facilitate much more rapid transfer to the FI schedule than to the RR schedule.
There is some evidence to support this view, which comes from the study reported by Wanchisen et al. (1989). In this experiment, rats that were transferred from a differential reinforcement of low-rate (DRL) to an FR schedule showed greater behavioral history effects than did rats that were transferred from an FR to a DRL schedule. This effect could be explained in that greater inhibitory control develops on a DRL schedule than on an FR schedule. Thus, the inhibitory control generated by the history DRL schedule would promote the pause-response pattern typical of the FR schedule. In contrast, the relatively weaker inhibitory control developed by the FR schedule would take longer to promote the slow rates necessary on a DRL schedule. Of course, this result is also consistent with a response-rate effect (the faster rates on the FR-history schedule would make it difficult to learn to respond on a DRL schedule). The final experiment sought to investigate the possibility that inhibitory control would facilitate transfer from ratio to FI schedules.

\section{Method}

Subjects and Apparatus. Eight experimentally naive male Lister hooded rats participated in Experiment 3. All of the rats were from 4 to 5 months old at the start of training, had a free-feeding body-weight range of $355-400 \mathrm{~g}$, and were maintained at $85 \%$ of this weight throughout the experiment. The subjects were housed as was described in Experiment 1. The apparatus was the same as that described in Experiment 1, with the exception that both levers were permanently inserted in the conditioning chamber.

Procedure. All rats were magazine and leverpress trained as described in Experiment 2. Following this initial training, rats were all exposed to a multiple RR-VR schedule (half with the RR schedule on the right lever, and half with the VR schedule on the left lever). The RR schedule was programmed as described previously. The VR component was programmed to have the same mean value as the RR schedule but was composed of a number of discrete ratio values selected randomly for each reinforcement. These values had a mean of the RR value and a range as specified. The components of the multiple schedule were presented in alternation. The ratio criterion value was increased during this period as follows. Sessions 3-4, RR-5 VR-5 (range 3-7); Sessions 5-6, RR-10 VR-10 (range 5-15); Sessions 7-8, RR-15 VR-15 (range 7-22); Sessions 9-10, RR-20 VR-20 (range 10-30); Sessions 11-12, RR-25 VR-25 (range 15-35); Sessions 13-20, RR-30 VR-30 (range 20-40); Sessions 21-30, RR-40 VR-40 (range 20-60); Sessions 31-40, RR-50 VR-50 (range 20-80); and Sessions 41-60, RR-60 VR-60 (range 20-100). As noted above, each session comprised six 5-min exposures to the RR components and six 5-min exposures to the VR components, presented in strict alternation.

Following exposure to this pretraining, the rats were all transferred to a multiple FI 60 -sec FI 60 -sec schedule. This training continued for 10 sessions. During each session, there were twelve 5-min components - six to each lever as described above - and the stimuli associated with the levers were the same as those in the history phase of training.

\section{Results and Discussion}

Following the first phase of training with the RR and VR schedules, the mean total number of responses emitted during the final session for the RR component was 2,895 (96.5 responses/min), and this score was 2,892 (96.4 responses/min) for the VR component.

The top panel of Figure 3 shows the mean responses emitted for each component during each successive 6-sec 

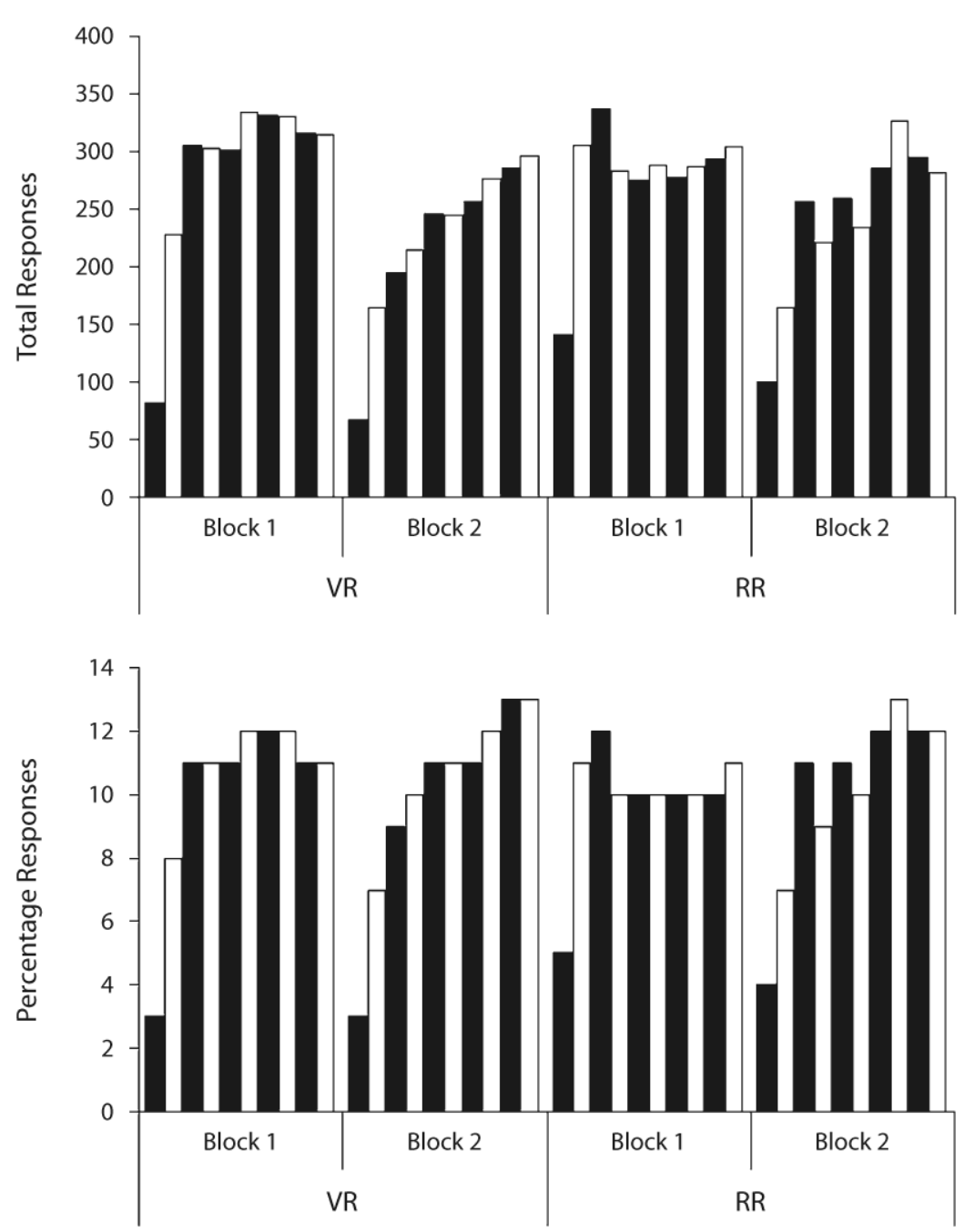

Figure 3. Results from Experiment 3. Top panel: Mean number of responses emitted in each 6-sec interval during exposure to the multiple FI 60-sec FI 60-sec schedule over the four 5-session blocks of training. Bottom panel: Mean percentage of total responses emitted during each successive 6-sec interval of the multiple FI 60-sec FI 60-sec schedule. $R R$, prior training on a random ratio schedule; VR, prior training on a variable ratio schedule.

interval of the multiple FI-FI schedule over the two 5 -session blocks of training. These data are replotted as percentages of the total responses emitted in each 6-sec interval for the two components in the right panel of Figure 3 . The response data (top panel) were analyzed by a three-factor repeated measures ANOVA (schedule $\times$ session $\times$ interval). The data and statistical analysis show that there was no difference in the rate of response between schedules (main effect schedule, $F<1$ ). Responding did not differ over the course of training (main effect session, $p>.05$ ), and responding altered across the intervals [main effect interval, $F(9,63)=264.65]$. An inspection of these data also shows a variety of interactions between the components across the intervals, which changed over the course of training [three-way interaction, $F(9,63)=8.77$ ] .

The three-way interaction was analyzed by a series of two-factor ANOVAs (component $\times$ interval) conducted on each session. Inspection and analyses of the data for Sessions 1-5 show that there was no difference between the responding due to pretraining $(F<1)$, that there were differences across the intervals $[F(9,63)=117.66]$, and that the pattern across the intervals differed between the components $[F(9,63)=10.35]$. Both components showed a similar pattern of a low rate immediately after reinforcement, followed by a fairly constant high rate across the interval. However, this effect was much more pronounced in the RR component than in the VR component. The RR component tended to have more responses in the first interval than did the former condition $[t(7)=10.95]$.

During the second 5-session block of training on the FI schedule, there were no differences between the responding due to pretraining $(F<1)$, there were differences in responding across the intervals $[F(9,63)=175.39]$, and the pattern of responding across the intervals differed 
between the components $[F(9,63)=9.89]$. Both components now showed a gradually increasing trend across the session, but, if anything, there were more responses emitted in the first interval in the previous RR component than there were in the previous VR component. This result was not statistically significant $(p>.05)$, however.

These data show that there were differences between the patterns of responding maintained on an FI schedule after training on VR and RR schedules. This pattern was not the result of differences in rates of response per se, since both groups had similar rates at the start of training on the FI schedule. It was also not due to differences in the rate of reinforcement, since both groups had the same rate of reinforcement in both pretraining and FI training. The difference is possibly due to the inhibitory effect of reinforcement on subsequent responding developed on the VR schedule, as compared with the RR schedule. The former schedule was programmed never to reinforce responding immediately following responding, whereas this could happen with a .017 probability in the RR schedule. That the effect was rather transient suggests that a difference between the schedules in this regard was slight and did not stand against the current contingency for long. Nevertheless, it provides corroboration for the suggestion that history effects - when transferring to an FI schedule - depend on the history of inhibitory control on the previous schedule.

\section{GENERAL DISCUSSION}

The present series of experiments examined the influence of prior ratio and interval training on the subsequent development of FI patterns of response. The present results showed transient effects of prior training on ratio schedules on subsequent FI performance. These transient effects were similar to those noted by Freeman and Lattal (1992). They were also in the same direction as were those reported by Wanchisen et al. (1989), but of more limited duration. Thus, these results support hypotheses focusing on the discrete retarding effect of prior ratio training that occurs uniquely to the facilitative effect of prior schedules with temporal control.

A novel finding from the present series of experiments relates to the retarding effect of exposure to an RI schedule on subsequent FI performance. Although technically an interval schedule, patterns of response that are characteristic of RI pretraining resulted in a comparatively slow development of postreinforcement pauses that are characteristic of FI scheduled response. This effect was shown to persist both within and between subjects for Blocks 1 and 2 (Sessions $1-5 ; 6-10$ ), and thus is unlikely to be due to effects produced by the yoked-controls procedure (see Church, 1964).

The fact that RI schedules produce a stronger retardation in the development of FI responding than do RR schedules could be explained in a number of ways. As was previously mentioned, possibilities such as the rate of reinforcement prior to transfer to the FI schedule were ruled out by use of a yoking procedure. However, response-rate differences and differences in the nature of the schedule control were alternative possibilities. That differences were found in the effects of prior exposure to RR and VR schedules on FI schedule performance suggests that inhibitory control may have some role to play in these effects. Although this does, to some degree, reflect the temporal-based hypothesis of Trapold et al. (1965), the fact that disruption also occurred in comparison with RR and VR schedules suggests that temporal control cannot be the sole factor in the facilitation of transfer between schedules. Additionally, note that in some schedules (e.g., VI), the delivery of reinforcement does not signal the subsequent absence of reinforcement for a period of time in the way it does to some extent in others (e.g., RR, VR). In fact, this effect is notably stronger in VR than in RR schedules, since the former is specifically programmed not to reinforce responding for a certain number of responses following reinforcement.

In this regard, VR schedules can be considered similar to FR schedules. When transferring from schedules that allow some inhibitory control to develop by the reinforcer, transfer strongly develops on FI schedules (Dews, 1962). As such, delivery of a reinforcer is associated with a subsequent lack of reinforcement, resulting in the inhibition of immediately proceeding behavior that may facilitate the emergence of the typical pattern of responding on FI schedules. Certainly, the present data are consistent with two earlier investigations of history effects. Cole (2001) noted better transfer to FI patterns when DRL rather than FR schedules were used. Similarly, FR schedules seem to produce better transfer than do VR schedules (cf. Baron \& Leibenweber, 1995; Freeman \& Lattal, 1992). Moreover, Wanchisen et al. (1989) noted stronger history effects when transferring from a schedule with strong inhibitory control (DRL) to one with weak inhibitory control (FR) in the development of pausing following reinforcement than the other way around.

These data go some way to resolving the apparent pattern of discrepant results seen in the literature and suggest a straightforward explanation of the history effects seen when transferring onto FI schedules. As per temporal hypotheses, when transferring to a schedule that characteristically produces a pause following reinforcement, schedules that have stronger inhibitory control achieve transfer more rapidly than those that do not.

\section{AUTHOR NOTE}

This research was funded by a grant from The Mechner Foundation, U.S.A., to P.R. Some of these data were submitted in partial fulfillment of an M.Phil. degree by T.A.M. We thank the School of Psychology, University of Leeds, who made facilities available for the conduct of this work, and Gillian Cardwell for her help with the collection of some of these data and for the care of the animals. We also thank Jim Wright, Lisa A. Osborne, Adam Doughty, and Andy Lattal for their support and comments on this research. Correspondence concerning this article should be addressed to P. Reed, Department of Psychology, Swansea University, Singleton Park, Swansea SA2 8PP, Wales (e-mail: p.reed@swansea.ac.uk).

Note-This article was accepted by the previous editorial team, when Shepard Siegel was Editor

\section{REFERENCES}

Baron, A., \& Leibenweber, A. (1995). Effects of a variable-ratio conditioning history on sensitivity to fixed-interval contingencies in rats Journal of the Experimental Analysis of Behavior, 63, 97-110. 
Church, R. M. (1964). Systematic effect of random error in the yoked control design. Psychological Bulletin, 62, 122-131.

Cole, M. R. (2001). The long-term effect of high- and low-rate responding on fixed-interval responding in rats. Journal of the Experimental Analysis of Behavior, 75, 43-54.

Dembo, M., Becerra de Penfold, J., Ruiz, R., \& Casalta, H. (1985). Properties of behavior under different random ratio and random interval schedules: A parametric study. Behavioural Processes, 10, 241-248.

Dews, P. B. (1962). The effect of multiple S-super(!D) periods on responding on a fixed-interval schedule. Journal of the Experimental Analysis of Behavior, 5, 369.

Dews, P. B. (1978). Studies on responding under fixed-interval schedules of reinforcement: II. The scalloped pattern of the cumulative record. Journal of the Experimental Analysis of Behavior, 29, 65-75.

Doughty, A., Reed, P., \& Lattal, K. A. (2004). Differential reinstatement predicted by preextinction response rate. Psychonomic Bulletin \& Review, 11, 1118-1123.

Ferster, C. B., \& Skinner, B. F. (1957). Schedules of reinforcement. New York: Appleton-Century-Crofts.

Freeman, T. J., \& LatTAL, K. A. (1992). Stimulus control of behavioral history. Journal of Experimental Analysis of Behavior, 57, 5-15.

Howell, D. C. (1997). Statistical methods for psychology. New York: Duxbury.

Keenan, M., \& Leslie, J. C. (1986). Varying response-reinforcer contiguity in a recycling conjunctive schedule. Journal of the Experimental Analysis of Behavior, 45, 317-332.

Lopez, F., \& Menez, M. (2005). Effects of reinforcement history on response rate and response pattern in periodic reinforcement. Journal of the Experimental Analysis of Behavior, 83, 221-241.

Mechner, F., Hyten, C., Field, D. P., \& Madden, G. J. (1997). Using revealed operants to study the structure and properties of human operant behavior. Psychological Record, 47, 45-68.

Mechner, F., \& Jones, L. (2003). Learning history and resurgence patterns. Retrieved June 24, 2006, from www.mechnerfoundation .org/revop.doc.
Reed, P., \& Morgan, T. A. (2006). Resurgence of response sequences during extinction in rats shows a primacy effect. Journal of the Experimental Analysis of Behavior, 86, 307-315.

Reed, P., \& Morgan, T. A. (2007). Resurgence of behavior during extinction depends on previous rate of response. Learning \& Behavior, 35, 106-114.

Roberts, J. E., TARPy, R. M., \& Lea, S. E. G. (1984). Stimulus-response overshadowing: Effects of signaled reward on instrumental responding as measured by response rate and resistance to change. Journal of Experimental Psychology: Animal Behavior Processes, 10, 244-255.

TATHAM, T. A., \& Wanchisen, B. A. (1998). Behavioral history: A definition and some common findings from two areas of research. Behavior Analyst, 21, 241-251.

ToAL, L., \& LeSLIE, J. C. (1984). Temporal control by signals of interval duration within variable-interval schedules. Journal of the Experimental Analysis of Behavior, 42, 267-277.

Trapold, M. A., Carlson, J. G., \& Myers, W. A. (1965). The effect of noncontingent fixed- and variable-interval reinforcement upon subsequent acquisition of the fixed-interval scallop. Psychonomic Science, 2, 261-262.

Wanchisen, B. A., Tatham, T. A., \& Mooney, S. E. (1989). Variableratio conditioning history produces high- and low-rate fixedinterval performance in rats. Journal of the Experimental Analysis of Behavior, 52, 167-179.

WEINER, H. (1964). Conditioning history and human fixed-interval performance. Journal of the Experimental Analysis of Behavior, 7, 383-385

WeINER, H. (1969). Controlling human fixed-interval performance. Journal of the Experimental Analysis of Behavior, 12, 349-373.

ZeILER, M. D. (1977). Schedules of reinforcement: The controlling variables. In W. K. Honig \& J. E. R. Staddon (Eds.), Handbook of operant behavior (pp. 201-232). New York: Prentice Hall.

(Manuscript received June 2, 2007; revision accepted for publication October 31, 2007.) 